\title{
Enumeration of S-Motzkin paths from left to right and from right to left: a kernel method approach
}

\author{
Helmut Prodinger \\ Department of Mathematics \\ University of Stellenbosch \\ 7602 Stellenbosch, South Africa \\ email: hproding@sun.ac.za
}

(Received: October 23, 2019, and in revised form September 30, 2020.)

\begin{abstract}
The area of S-Motzkin paths (bijective to ternary trees) is calculated using the kernel method by enumerating these (partial) paths with fixed end-point resp. starting point.
\end{abstract}

Mathematics Subject Classification(2010). 05A15.

Keywords: ternary tree, S-Motzkin path, kernel method, generalised binomial series.

\section{Introduction}

The area of a lattice path $\mathscr{A}$ is defined to be $\sum_{i>0} h_{i}(\mathscr{A})$ where $h_{i}(\mathscr{A})$ is the height of the path $\mathscr{A}$ at $x$-coordinate $i$. This parameter has been studied in various types of paths [8, 9, 1]. In this paper, the area will be available as a corollary of the enumeration of partial (incomplete) families of lattice paths that are bijective to ternary trees, and thus enumerated by the numbers $\frac{1}{2 n+1}\left(\begin{array}{c}3 n \\ n\end{array}\right)$.

We study such a family that was recently introduced by [11, and find explicit formulae for them ending after $n$ steps at level $k$, both, from left to right (starting at the origin) and also from right to left (starting at the end and going backwards). The latter instance is the more challenging one.

S-Motzkin paths can be transformed into other more traditional ternary objects, like ternary trees and ternary paths. No use of this will, however, made here, to keep the discussion self-contained. This is also beneficial from a pedagogic point of view, since it shows how to deal with a system of two equations and the kernel method, in the presence of cubic equations.

The enumeration in this paper involves a lattice path called an S-Motzkin path. This is a subclass of Motzkin paths introduced by the authors in a previous publication [11. For completeness, we provide the definition again.

DeFinition 1.1 An S-Motzkin path of length $3 n$ is

- a Motzkin path

- it consists of $n$ up-steps $(1,1), n$ down-steps $(1,-1), n$ level-steps $(1,0)$

- ignoring the down-steps, the level-steps and the up-steps alternate, starting with a level-step.

DOI: $10.1515 /$ puma-2015-0039

(C) 2020 Prodinger. This is an open access article licensed under the Creative Commons Attribution-NonCommercial-NoDerivs License (http://creativecommons.org/licenses/by-nc-nd/3.0/). 
We apply the kernel method to S-Motzkin paths as well as reverse S-Motzkin paths to obtain the area of S-Motzkin paths. As a bonus, we obtain the exact enumeration of partial S-Motzkin paths. It is particularly worthwhile to obtain results for this relatively new class of lattice paths, since, as seen in [7], this family appeared in a surprisingly unrelated context. It is perhaps of independent interest to note that S-Motzkin paths were introduced to solve a problem from a student olympiad about frog hops [10].

A state-of-the-art survey about lattice path enumeration is [6]; it does not include S-Motzkin paths, as they are new.

Our main findings are the enumerations of four classes of paths (defined later): (1), (2), (3), (4), and the area (5). Informally, these are the enumeration of (partial) S-Motzkin paths, ending on a prescribed level, both, when considering them from left-to-right and from right-to-left. The area of an S-Motzkin is the sum of all its ordinates. The sum of this is computed, when summing over all S-Motzkin paths of the same length $3 n$.

\section{Preliminary computations}

The following computation appears frequently in this paper, and we want to do it only once: Here, $x=t(1-t)^{2}$, and in all applications we will have $z^{3}=x$.

$$
\begin{aligned}
{\left[x^{m}\right] \frac{1}{(1-t)^{j}} } & =\frac{1}{2 \pi i} \oint \frac{d x}{x^{m+1}} \frac{1}{(1-t)^{j}} \\
& =\frac{1}{2 \pi i} \oint \frac{d t(1-3 t)(1-t)}{t^{m+1}(1-t)^{2 m+2}} \frac{1}{(1-t)^{j}} \\
& =\left[t^{m}\right] \frac{1-3 t}{(1-t)^{2 m+j+1}}=\left(\begin{array}{c}
3 m+j \\
m
\end{array}\right)-3\left(\begin{array}{c}
3 m+j-1 \\
m-1
\end{array}\right) .
\end{aligned}
$$

The binomial series notation as given in [5]

$$
\mathscr{B}_{t}(x)^{r}=\sum_{k \geq 0}\left(\begin{array}{c}
t k+r \\
k
\end{array}\right) \frac{r}{t k+r} x^{k}
$$

can often be used to express certain quantities that appear in this paper. This is elegant, but the notation using the variable $t$ (as in $x=t(1-t)^{2}$ ) seems to be more efficient. Note that the coefficient of $x^{n}$ in $\mathscr{B}_{3}(x)^{1}$ is $\frac{1}{3 n+1}\left(\begin{array}{c}3 n+1 \\ n\end{array}\right)=\frac{1}{2 n+1}\left(\begin{array}{c}3 n \\ n\end{array}\right)$, which is the number of ternary trees of size $n$.

The generating function for ternary trees and also S-Motzkin paths and ternary paths can be rewritten as $\mathscr{B}_{3}(x)$ using this notation. We will frequently use the substitution $x=t(1-t)^{2}$.

From the Lagrange inversion formula [2, Theorem A.2] we find

$$
\left[x^{n}\right] t^{k}=\frac{k}{n}\left[w^{n-k}\right] \frac{1}{(1-w)^{2 n}}=\frac{k}{n}\left(\begin{array}{c}
3 n-k-1 \\
n-k
\end{array}\right) \quad \Rightarrow \quad t^{k}=\sum_{n \geq k}\left(\begin{array}{c}
3 n-k-1 \\
n-k
\end{array}\right) \frac{k}{n} x^{n} .
$$

Using this we can compute an expansion that is useful in the context of ternary paths and variants:

$$
\sqrt{4 t-3 t^{2}}=2 t^{1 / 2} \sqrt{1-\frac{3}{4} t}=2 t^{1 / 2} \sum_{k \geq 0}(-1)^{k}\left(\frac{3}{4}\right)^{k}\left(\begin{array}{l}
\frac{1}{2} \\
k
\end{array}\right) t^{k}
$$




$$
\begin{aligned}
& =2 t^{1 / 2} \sum_{k \geq 0}(-1)^{k}\left(\frac{3}{4}\right)^{k}\left(\begin{array}{c}
\frac{1}{2} \\
k
\end{array}\right) \sum_{n \geq k}\left(\begin{array}{c}
3 n-k-1 \\
n-k
\end{array}\right) \frac{k}{n} x^{n} \\
& =2 t^{1 / 2} \sum_{n \geq 0} x^{n} \sum_{1 \leq k \leq n}(-1)^{k}\left(\frac{3}{4}\right)^{k}\left(\begin{array}{c}
\frac{1}{2} \\
k
\end{array}\right)\left(\begin{array}{c}
3 n-k-1 \\
n-k
\end{array}\right) \frac{k}{n} \\
& =-2 t^{1 / 2} \sum_{n \geq 0}\left(\begin{array}{c}
3 n-\frac{3}{2} \\
2 n
\end{array}\right) \frac{1}{2 n-1} x^{n} .
\end{aligned}
$$

The simplification of the inner sum was done by a computer.

This is one way to switch between expressions in the variable $t$ and expressions in terms of the binomial series notation.

\section{The enumeration of partial S-Motzkin paths}

\subsection{S-Motzkin paths}

A partial S-Motzkin path is a Motzkin path that can be continued to be an S-Motzkin path, or we might say the first part (of length $m$, say) of an existing S-Motzkin path. Since S-Motzkin paths are not symmetric w. r. t. left vs. right, we will later also consider this concept from right to left (which turns out to be more difficult).

Let $a_{n, k}$ denote the number of partial S-Motzkin path of length $n$ which ends at height $k$ and the last step of the path from the step set $\{(1,0),(1,1)\}$ is a $(1,1)$ step. Similarly, let $b_{n, k}$ denote the number of partial S-Motzkin path of length $n$ which ends at height $k$ and the last step of the path from the step set $\{(1,0),(1,1)\}$ is a $(1,0)$ step. It is convenient to set $a_{0,0}=1$ and $b_{0,0}=0$.

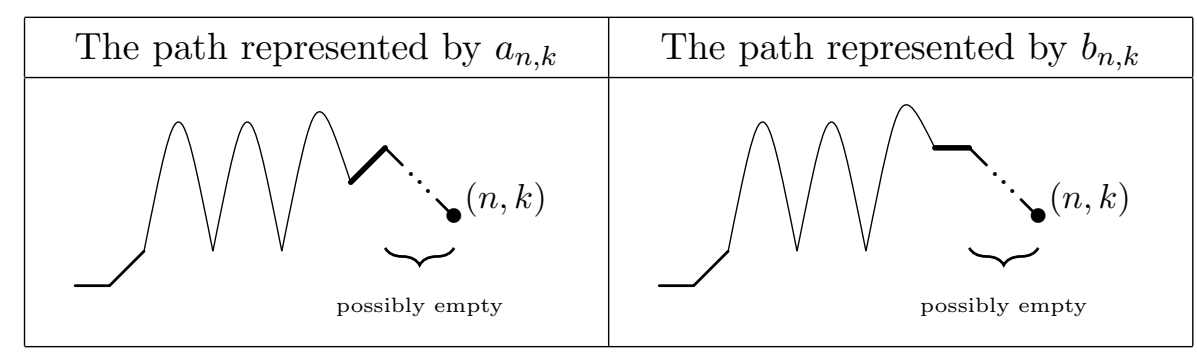

It is easily seen that the following recurrence relations hold:

$$
\begin{aligned}
a_{n, k} & =b_{n-1, k-1}+a_{n-1, k+1}, \\
b_{n, k} & =a_{n-1, k}+b_{n-1, k+1} .
\end{aligned}
$$

These recurrence relations are valid for $n \geq 1$ and $k \geq 0$; the quantity $b_{n-1,-1}$ must be interpreted as 0 . We let

$$
A(z, u)=\sum_{n \geq 0} \sum_{k \geq 0} a_{n, k} z^{n} u^{k} \quad \text { and } \quad B(z, u)=\sum_{n \geq 0} \sum_{k \geq 0} b_{n, k} z^{n} u^{k}
$$


and sum over $n$ and $k$ to obtain the following system of equations

$$
\begin{aligned}
A(z, u)-1 & =z u B(z, u)+\frac{z}{u} A(z, u)-\frac{z}{u} A(z, 0), \\
B(z, u) & =z A(z, u)+\frac{z}{u} B(z, u)-\frac{z}{u} B(z, 0) .
\end{aligned}
$$

Solving the system of equations for $A(z, u)$ and $B(z, u)$ gives

$$
\begin{aligned}
& A(z, u)=\frac{-u^{2}+z u A(z, 0)+z u-z^{2} A(z, 0)+B(z, 0) z^{2} u^{2}}{z^{2} u^{3}-u^{2}+2 z u-z^{2}}, \\
& B(z, u)=\frac{z\left(-u^{2}+u B(z, 0)+z u A(z, 0)-z B(z, 0)\right)}{z^{2} u^{3}-u^{2}+2 z u-z^{2}} .
\end{aligned}
$$

The polynomial in the denominator, $z^{2} u^{3}-u^{2}+2 z u-z^{2}$, is of interest to us. Using the substitution $u=z w$ along with $z^{3}=t(1-t)^{2}$, we obtain

$$
\left(t^{2} w^{2}-2 t w^{2}+t w+w^{2}-2 w+1\right)(t w-1)=0 .
$$

Therefore the three roots (expressed again in the variable $u$ ) are given by

$$
v_{1}=\frac{z}{t}, \quad v_{2}=-z \frac{t-2+\sqrt{4 t-3 t^{2}}}{2(1-t)^{2}}, \quad v_{3}=-z \frac{t-2-\sqrt{4 t-3 t^{2}}}{2(1-t)^{2}} .
$$

Alternatively, the three roots can be written using $\mathscr{B}_{3}\left(z^{3}\right)$ and $\mathscr{B}_{3 / 2}\left( \pm z^{3 / 2}\right)$, but this will not be used.

The roots can also be expressed as series (Puiseux series, to be exact)

$$
\begin{aligned}
& v_{1}=z^{-2}-2 \sum_{n \geq 0} \frac{(3 n) !}{(2 n) !(n+1) !} z^{3 n+1}, \\
& v_{2}=-6 \sum_{n \geq 0} \frac{(6 n+1) !(n+1) !}{(3 n) !(2 n+3) !(2 n) ! 2^{4 n}} z^{3 n+5 / 2}+\sum_{n \geq 0} \frac{(3 n) !}{(2 n) !(n+1) !} z^{3 n+1}, \\
& v_{3}=6 \sum_{n \geq 0} \frac{(6 n+1) !(n+1) !}{(3 n) !(2 n+3) !(2 n) ! 2^{4 n}} z^{3 n+5 / 2}+\sum_{n \geq 0} \frac{(3 n) !}{(2 n) !(n+1) !} z^{3 n+1},
\end{aligned}
$$

and it can be shown that these series are indeed the roots by converting them to hypergeometric functions and using Clausen's identity [12], but we mention this just for interest and will not use it further. Gessel and Xin [3] used such an approach, but it is a tour the force and fortunately, here, we can avoid to go that route.

Note that

$$
v_{2}+v_{3}=-\frac{z(t-2)}{(t-1)^{2}} \quad \text { and } \quad v_{2} v_{3}=\frac{z^{2}}{(t-1)^{2}} .
$$

We know that $A(z, u)$ and $B(z, u)$ have power series expansions around $(0,0)$, so the factors $\left(u-v_{2}\right)$ and $\left(u-v_{3}\right)$ in the denominator must also be factors in the numerator. Hence we can find $A(z, 0)$ and $B(z, 0)$ by solving the system

$$
\begin{aligned}
& 0=-v_{2}^{2}+z v_{2} A(z, 0)+z v_{2}-z^{2} A(z, 0)+B(z, 0) z^{2} v_{2}^{2}, \\
& 0=-v_{3}^{2}+v_{3} B(z, 0)+z v_{3} A(z, 0)-z B(z, 0),
\end{aligned}
$$


to obtain

$$
A(z, 0)=-\frac{v_{2} v_{3}}{z^{2}}+\frac{v_{2}}{z}+\frac{v_{3}}{z} \quad \text { and } \quad B(z, 0)=\frac{v_{2} v_{3}}{z} .
$$

Substituting these back into the original equations yields

$$
\begin{aligned}
& A(z, u)=\frac{u^{2} v_{2} v_{3} z^{2}-u v_{2} v_{3}-u^{2} z+u v_{2} z+u v_{3} z+v_{2} v_{3} z+u z^{2}-v_{2} z^{2}-v_{3} z^{2}}{\left(u-v_{1}\right)\left(u-v_{2}\right)\left(u-v_{3}\right) z^{3}}, \\
& B(z, u)=-\frac{1}{\left(u-v_{1}\right) z} .
\end{aligned}
$$

Since we know that $\left(u-v_{2}\right)$ and $\left(u-v_{3}\right)$ are factors of the numerator of $A(z, u)$ we can simplify $A(z, u)$ by dividing these two factors out (we consistently use the variable $t$ for that). After simplification,

$$
A(z, u)=\frac{1}{1-t} \frac{1}{1-\frac{t u}{z}} \quad \text { and } \quad B(z, u)=\frac{t}{z^{2}} \frac{1}{1-\frac{t u}{z}} .
$$

Extraction of coefficients is now easy:

$$
\left[u^{k}\right] A(z, u)=\frac{1}{1-t} \frac{t^{k}}{z^{k}}, \quad\left[u^{k}\right] B(z, u)=\frac{t^{k+1}}{z^{k+2}}
$$

Furthermore

$$
\left[z^{n} u^{k}\right] A(z, u)=\left[z^{n+k}\right] t^{k}
$$

These coefficients are 0 unless $n+k=3 m$ for some $m \in \mathbb{N}$. Thus we will compute the coefficient of $z^{3 m-k}$ in $\left[u^{k}\right] A(z, u)$, and we write $x=z^{3}$ for convenience, as before.

$$
\begin{aligned}
{\left[z^{3 m-k} u^{k}\right] A(z, u) } & =\left[z^{3 m}\right] \frac{1}{1-t} t^{k}=\left[x^{m}\right] \frac{1}{1-t} t^{k} \\
& =\left[t^{m-k}\right] \frac{1-3 t}{(1-t)^{2 m+2}} \\
& =\left(\begin{array}{c}
3 m-k+1 \\
m-k
\end{array}\right)-3\left(\begin{array}{c}
3 m-k \\
m-k-1
\end{array}\right) .
\end{aligned}
$$

Likewise,

$$
\left[z^{n} u^{k}\right] B(z, u)=\left[z^{n+k+2}\right] t^{k+1} .
$$

Since this only makes sense if $n+k+2=3 m$ for some $m \in \mathbb{N}$. Thus we read off the coefficient of $z^{3 m-k-2}$ :

$$
\begin{aligned}
{\left[z^{3 m-k-2} u^{k}\right] B(z, u) } & =\left[z^{3 m}\right] t^{k+1}=\left[x^{m}\right] t^{k+1}=\left[t^{m-k-1}\right] \frac{1-3 t}{(1-t)^{2 m+2}} \\
& =\left(\begin{array}{c}
3 m-k \\
m-k-1
\end{array}\right)-3\left(\begin{array}{c}
3 m-k-1 \\
m-k-2
\end{array}\right)
\end{aligned}
$$




\subsection{Reverse S-Motzkin paths}

A reverse S-Motzkin path is an S-Motzkin path read from right to left. Alternatively, we might say that a reverse S-Motzkin path is a Motzkin path of length $3 n$ with $n$ of each type of step such that the first step from the step set $\{(1,0),(1,-1)\}$ is a $(1,-1)$ step. Furthermore, $(1,0)$ and $(1,-1)$ steps alternate.

Let $c_{n, k}$ denote a partial reverse S-Motzkin path of length $n$ which ends at height $k$ and the last step of the path in the step set $\{(1,0),(1,-1)\}$ is a $(1,0)$ step. Similarly, let $d_{n, k}$ denote a partial reverse S-Motzkin path of length $n$ which ends at height $k$ and the last step of the path in the step set $\{(1,0),(1,-1)\}$ is a $(1,-1)$ step.

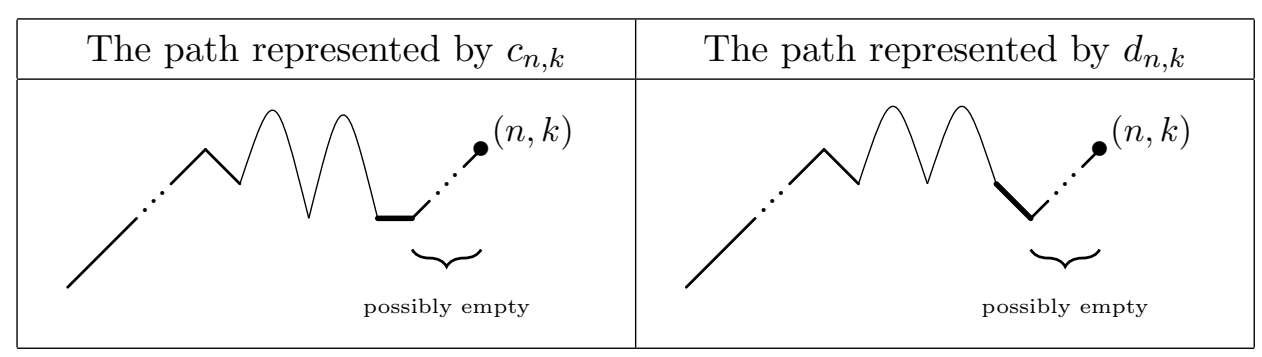

It is easily seen that the following recurrence relations hold:

$$
\begin{aligned}
c_{n, k} & =c_{n-1, k-1}+d_{n-1, k}, \\
d_{n, k} & =d_{n-1, k-1}+c_{n-1, k+1} .
\end{aligned}
$$

These recurrences hold for $n \geq 1$ and $k \geq 0 ; c_{n-1,-1}$ and $d_{n-1,-1}$ must be interpreted as zero, and the initial values are $c_{0,0}=1$ and $d_{0,0}=0$.

Let

$$
C(z, u)=\sum_{n \geq 0} \sum_{k \geq 0} c_{n, k} z^{n} u^{k} \quad \text { and } \quad D(z, u)=\sum_{n \geq 0} \sum_{k \geq 0} d_{n, k} z^{n} u^{k}
$$

and sum the recursion over $n$ and $k$. This results in

$$
\begin{aligned}
C(z, u)-1 & =z u C(z, u)+z D(z, u), \\
D(z, u) & =z u D(z, u)+\frac{z}{u} C(z, u)-\frac{z}{u} C(z, 0) .
\end{aligned}
$$

Solving this system gives

$$
C(z, u)=\frac{u-u^{2} z-C(z, 0) z^{2}}{z^{2} u^{3}-2 z u^{2}+u-z^{2}} \quad \text { and } \quad D(z, u)=\frac{C(z, 0) u z^{2}-C(z, 0) z+z}{z^{2} u^{3}-2 z u^{2}+u-z^{2}} .
$$

Note that the denominator is given by

$$
z^{2} u^{3}-2 z u^{2}+u-z^{2}
$$

whereas in the previous section $(\$ 3.1)$ the denominator was given by

$$
z^{2} u^{3}-u^{2}+2 z u-z^{2}=z^{2}\left(u-v_{1}\right)\left(u-v_{2}\right)\left(u-v_{3}\right) ;
$$


the explicit forms of the roots are repeated for convenience:

$$
v_{1}=\frac{z}{t}, \quad v_{2}=-z \frac{t-2+\sqrt{4 t-3 t^{2}}}{2(1-t)^{2}}, \quad v_{3}=-z \frac{t-2-\sqrt{4 t-3 t^{2}}}{2(1-t)^{2}} .
$$

The equation $z^{2} u^{3}-u^{2}+2 z u-z^{2}$ along with the substitution $u=1 / u$ and multiplication by $-u^{3}$ gives the denominator in the current case:

$$
-u^{3}\left(z^{2}\left(\frac{1}{u}\right)^{3}-\left(\frac{1}{u}\right)^{2}+2 z\left(\frac{1}{u}\right)-z^{2}\right)=-z^{2}+u-2 z u^{2}+z^{2} u^{3} .
$$

Therefore the roots of the polynomial $z^{2} u^{3}-2 z u^{2}+u-z^{2}$ are given by $v_{1}^{-1}, v_{2}^{-1}$, and $v_{3}^{-1}$, hence

$$
z^{2} u^{3}-2 z u^{2}+u-z^{2}=z^{2}\left(u-\frac{1}{v_{1}}\right)\left(u-\frac{1}{v_{2}}\right)\left(u-\frac{1}{v_{3}}\right) .
$$

Note that

$$
\frac{1}{v_{1}}=\frac{t}{z}, \quad \frac{1}{v_{2}}=\frac{-t+2+\sqrt{4 t-3 t^{2}}}{2 z}, \quad \frac{1}{v_{3}}=\frac{-t+2-\sqrt{4 t-3 t^{2}}}{2 z} .
$$

In the current right-to-left enumeration, $u-v_{1}^{-1}$ is the factor in the denominator that is also a factor of the numerator. Plugging in $u=v_{1}^{-1}$ into the numerator of $D(z, u)$ (the numerator of $C(z, u)$ could also be used) gives

$$
C(z, 0)=\frac{v_{1}}{v_{1}-z} .
$$

Using this value for $C(z, 0)$, it follows that

$$
\frac{C(z, 0) u z^{2}-C(z, 0) z+z}{u-v_{1}^{-1}}=\frac{z^{2} v_{1}}{v_{1}-z}=z^{2} C(z, 0),
$$

and thus

$$
D(z, u)=\frac{C(z, 0)}{\left(u-\frac{1}{v_{2}}\right)\left(u-\frac{1}{v_{3}}\right)}
$$

We can further write

$$
D(z, u)=\frac{1}{(1-t)\left(u-\frac{1}{v_{2}}\right)\left(u-\frac{1}{v_{3}}\right)},
$$

and representing this as a partial fraction gives

$$
D(z, u)=\frac{t}{z(1-t)} \frac{v_{3}}{\left(1-u v_{3}\right)\left(v_{3}-v_{2}\right)}-\frac{t}{z(1-t)} \frac{v_{2}}{\left(1-u v_{2}\right)\left(v_{3}-v_{2}\right)} .
$$

Therefore we can find the coefficients of $D(z, u)$ :

$$
\left[u^{k}\right] D(z, u)=\frac{t}{z(1-t)} \frac{v_{3}^{k+1}-v_{2}^{k+1}}{v_{3}-v_{2}} .
$$


The identity [4, eq. (22)] will be useful in calculating $\left[z^{n} u^{k}\right] D(z, u)$ and $\left[z^{n} u^{k}\right] C(z, u)$, so note that

$$
\begin{aligned}
\frac{v_{3}^{k+1}-v_{2}^{k+1}}{v_{3}-v_{2}} & =\sum_{i=0}^{\lfloor k / 2\rfloor}(-1)^{i}\left(\begin{array}{c}
k-i \\
i
\end{array}\right)\left(v_{2}+v_{3}\right)^{k-2 i}\left(v_{2} v_{3}\right)^{i} \\
& =\sum_{i=0}^{\lfloor k / 2\rfloor}(-1)^{i+k}\left(\begin{array}{c}
k-i \\
i
\end{array}\right) \frac{z^{k-2 i}(t-2)^{k-2 i}}{(t-1)^{2 k-4 i}} \frac{z^{2 i}}{(1-t)^{2 i}} \\
& =z^{k} \sum_{i=0}^{\lfloor k / 2\rfloor}(-1)^{i+k}\left(\begin{array}{c}
k-i \\
i
\end{array}\right) \frac{(t-2)^{k-2 i}}{(t-1)^{2 k-2 i}}
\end{aligned}
$$

Further,

$$
\left[u^{k}\right] D(z, u)=t z^{k-1} \sum_{i=0}^{\lfloor k / 2\rfloor}(-1)^{i+k-1}\left(\begin{array}{c}
k-i \\
i
\end{array}\right) \frac{(t-2)^{k-2 i}}{(t-1)^{2 k-2 i+1}}
$$

Now set $n=3 N+s-1, k=3 K+s$ for $s \in\{0,1,2\}$. Then

$$
\begin{aligned}
{\left[z^{n} u^{k}\right] D(z, u) } & =\left[z^{3 N+s-1} u^{3 K+s}\right] D(z, u) \\
& =\left[x^{N-K}\right] t \sum_{i=0}^{\lfloor k / 2\rfloor}(-1)^{i+k-1}\left(\begin{array}{c}
k-i \\
i
\end{array}\right) \frac{(t-2)^{k-2 i}}{(t-1)^{2 k-2 i+1}} \\
& =\left[x^{N-K}\right] t \sum_{i=0}^{\lfloor k / 2\rfloor} \sum_{j=0}^{k-2 i}(-1)^{i}\left(\begin{array}{c}
k-i \\
i
\end{array}\right)\left(\begin{array}{c}
k-2 i \\
j
\end{array}\right) \frac{1}{(1-t)^{2 k-2 i-j+1}} \\
& =\sum_{i=0}^{\lfloor k / 2\rfloor} \sum_{j=0}^{k-2 i}(-1)^{i}\left(\begin{array}{c}
k-i \\
i
\end{array}\right)\left(\begin{array}{c}
k-2 i \\
j
\end{array}\right) \\
& \times\left[\left(\begin{array}{c}
n+k-2 i-j+1 \\
(n-k+1) / 3-1
\end{array}\right)-3\left(\begin{array}{c}
n+k-2 i-j \\
(n-k+1) / 3-2
\end{array}\right)\right] .
\end{aligned}
$$

Similarly, for $C(z, u)$ we get

$$
\frac{u-u^{2} z-C(z, 0) z^{2}}{u-v_{1}^{-1}}=\frac{-\left(u v_{1} z-v_{1}+z\right)}{v_{1}}
$$

and thus

$$
C(z, u)=\frac{1-t-u z}{z^{2}\left(u-\frac{1}{v_{2}}\right)\left(u-\frac{1}{v_{3}}\right)}=\frac{(1-t-u z)}{(1-t)^{2}\left(1-v_{2} u\right)\left(1-v_{3} u\right)} .
$$

Rewriting $C(z, u)$ using partial fractions gives

$$
C(z, u)=\left[\frac{1}{1-t}-\frac{u z}{(1-t)^{2}}\right] \frac{1}{v_{3}-v_{2}}\left[\frac{v_{3}}{1-u v_{3}}-\frac{v_{2}}{1-u v_{2}}\right]
$$


which allows for coefficient extraction:

$$
\begin{aligned}
{\left[u^{k}\right] C(z, u)=} & \frac{1}{1-t} \frac{v_{3}^{k+1}-v_{2}^{k+1}}{v_{3}-v_{2}}-\frac{z}{(1-t)^{2}} \frac{v_{3}^{k}-v_{2}^{k}}{v_{3}-v_{2}} \\
= & z^{k} \sum_{i=0}^{\lfloor k / 2\rfloor}(-1)^{i+k}\left(\begin{array}{c}
k-i \\
i
\end{array}\right) \frac{(t-2)^{k-2 i}}{(t-1)^{2 k-2 i+1}} \\
& \quad-z^{k} \sum_{i=0}^{\lfloor(k-1) / 2\rfloor}(-1)^{i+k-1}\left(\begin{array}{c}
k-1-i \\
i
\end{array}\right) \frac{(t-2)^{k-1-2 i}}{(t-1)^{2 k-2 i}} \\
= & z^{k} \sum_{i=0}^{\lfloor k / 2\rfloor} \sum_{j=0}^{k-2 i}(-1)^{i-1}\left(\begin{array}{c}
k-i \\
i
\end{array}\right)\left(\begin{array}{c}
k-2 i \\
j
\end{array}\right) \frac{1}{(1-t)^{2 k-2 i-j+1}} \\
& \quad-z^{k} \sum_{i=0}^{\lfloor(k-1) / 2\rfloor} \sum_{j=0}^{k-1-i}(-1)^{i-1}\left(\begin{array}{c}
k-i-1 \\
i
\end{array}\right)\left(\begin{array}{c}
k-1-2 i \\
j
\end{array}\right) \frac{1}{(1-t)^{2 k-2 i-j}} .
\end{aligned}
$$

Furthermore, with $n=3 N+s, k=3 K+s$,

$$
\begin{aligned}
& {\left[z^{n} u^{k}\right] C(z, u)=\left[x^{N-K}\right] \sum_{i=0}^{\lfloor k / 2\rfloor} \sum_{j=0}^{k-2 i}(-1)^{i}\left(\begin{array}{c}
k-i \\
i
\end{array}\right)\left(\begin{array}{c}
k-2 i \\
j
\end{array}\right) \frac{1}{(1-t)^{2 k-2 i-j+1}}} \\
& -\left[x^{N-K}\right] \sum_{i=0}^{\lfloor(k-1) / 2\rfloor} \sum_{j=0}^{k-1-i}(-1)^{i}\left(\begin{array}{c}
k-1-i \\
i
\end{array}\right)\left(\begin{array}{c}
k-1-2 i \\
j
\end{array}\right) \frac{1}{(1-t)^{2 k-2 i-j}} \\
& =\sum_{i=0}^{\lfloor k / 2\rfloor} \sum_{j=0}^{k-2 i}(-1)^{i}\left(\begin{array}{c}
k-i \\
i
\end{array}\right)\left(\begin{array}{c}
k-2 i \\
j
\end{array}\right) \\
& \times\left[\left(\begin{array}{c}
n+k-2 i-j+1 \\
(n-k) / 3
\end{array}\right)-3\left(\begin{array}{c}
n+k-2 i-j \\
(n-k) / 3-1
\end{array}\right)\right] \\
& -\sum_{i=0}^{\lfloor(k-1) / 2\rfloor} \sum_{j=0}^{k-1-i}(-1)^{i}\left(\begin{array}{c}
k-1-i \\
i
\end{array}\right)\left(\begin{array}{c}
k-1-2 i \\
j
\end{array}\right) \\
& \times\left[\left(\begin{array}{c}
n+k-2 i-j \\
(n-k) / 3
\end{array}\right)-3\left(\begin{array}{c}
n+k-2 i-j-1 \\
(n-k) / 3-1
\end{array}\right)\right] .
\end{aligned}
$$

\subsection{The area of S-Motzkin paths}

Consider an arbitrary S-Motzkin path of length $3 n$. This path can be decomposed at any height $k$ that the path attains into either an (1) $a_{\ell, k}$ path followed by a $c_{3 n-\ell, k}$ path or (2) a $b_{\ell, k}$ path followed by a $d_{3 n-\ell, k}$ path.

We see that the generating function for the total area (all the areas, summed over all S-Motzkin paths of the same length) is given by

$$
\sum_{k \geq 0} k\left(\left[u^{k}\right] A(z, u) \cdot\left[u^{k}\right] C(z, u)+\left[u^{k}\right] B(z, u) \cdot\left[u^{k}\right] D(z, u)\right) .
$$




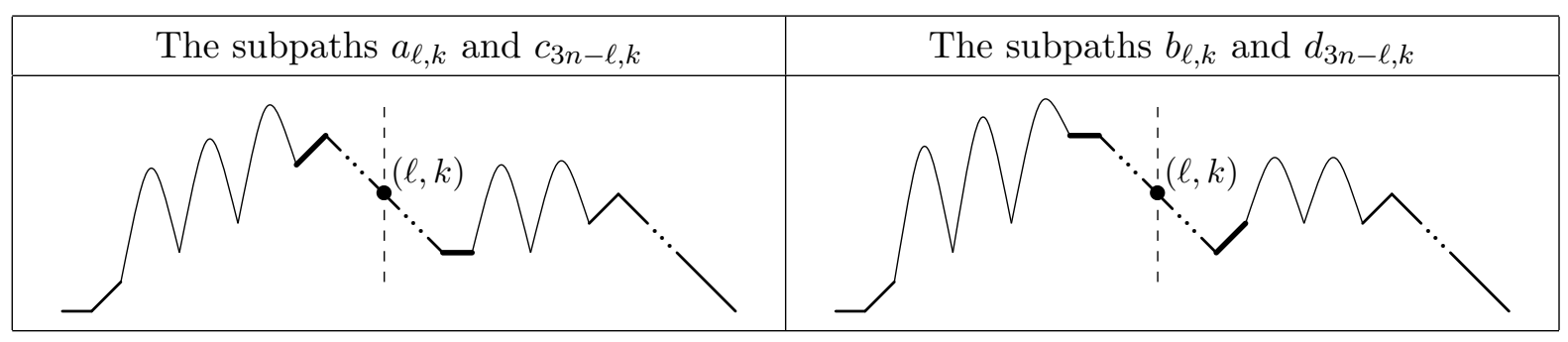

Fortunately, we have explicit forms for $\left[u^{k}\right] A(z, u),\left[u^{k}\right] B(z, u),\left[u^{k}\right] C(z, u),\left[u^{k}\right] D(z, u)$, and we compute the sum, using the forms of $v_{2}$ and $v_{3}$ involving $t$ (using a computer, of course):

$$
\begin{aligned}
& \sum_{k \geq 0} k\left(\left[u^{k}\right] A(z, u) \cdot\left[u^{k}\right] C(z, u)+\left[u^{k}\right] B(z, u) \cdot\left[u^{k}\right] D(z, u)\right) \\
& =\sum_{k \geq 0} k\left(\left(\frac{1}{z v_{2}^{k+1} v_{3}^{k+1}}-\frac{1}{z v_{2}^{k+2} v_{3}^{k+2}}\right) \cdot\left(\left(v_{3}^{k+1}-v_{2}^{k+1}\right)\left(\frac{v_{2} v_{3}-v_{2}^{2} v_{3}^{2} z}{z^{2}\left(v_{3}-v_{2}\right)}\right)+\left(v_{2}^{k}-v_{3}^{k}\right)\left(\frac{v_{2} v_{3}}{z\left(v_{3}-v_{2}\right)}\right)\right)\right. \\
& \left.\quad+\left(\frac{1}{z v_{2}^{k+1} v_{3}^{k+1}}\right) \cdot\left(v_{3}^{k+1}-v_{2}^{k+1}\right)\left(\frac{v_{2} v_{3}}{\left(v_{3}-v_{2}\right)\left(1-z v_{2} v_{3}\right)}\right)\right)=\frac{t}{(1-t)^{2}(1-3 t)^{2}} .
\end{aligned}
$$

Using Cauchy's integral formula we find that the area of S-Motzkin paths of length $3 n$ is given by

$$
\begin{aligned}
{\left[x^{n}\right] \frac{t}{(1-t)^{2}(1-3 t)^{2}} } & =\frac{1}{2 \pi i} \oint \frac{d x}{x^{n+1}} \frac{t}{(1-t)^{2}(1-3 t)^{2}} \\
& =\frac{1}{2 \pi i} \oint \frac{d t}{t^{n}(1-t)^{2 n+3}} \frac{1}{(1-3 t)} \\
& =\left[t^{n-1}\right] \frac{1}{(1-t)^{2 n+3}} \frac{1}{(1-3 t)} \\
& =\sum_{k=0}^{n-1} 3^{k}\left[t^{n-1-k}\right] \frac{1}{(1-t)^{2 n+3}} \\
& =\sum_{k=0}^{n-1} 3^{k}\left(\begin{array}{c}
3 n+1-k \\
n-1-k
\end{array}\right) .
\end{aligned}
$$

\section{Acknowledgments}

B. Hackl, C. Heuberger, S. Selkirk, and S. Wagner contributed at various stages to this project. Their contributions are gratefully acknowledged.

The insightful comments of one reviewer are also gratefully acknowledged.

\section{References}

[1] C. Banderier And B. Gittenberger, Analytic combinatorics of lattice paths: enumeration and asymptotics for the area, Discrete Math. Theor. Comput. Sci. Proc., AG (2006) 345-355. 
[2] P. Flajolet and R. Sedgewick, Analytic Combinatorics, Cambridge University Press, 2009.

[3] I. M. Gessel And G. XIn, The generating function of ternary trees and continued fractions, Electron. J. Combin., 13 (2006) \#R53, 48 pp.

[4] H. Gould, The Girard-Waring power sum formulas for symmetric functions and Fibonacci sequences, Fibonacci Quart., 37 (1999) 135-140.

[5] R. L. Graham, D. E. Knuth and O. Patashnik, Concrete Mathematics, Addison-Wesley, Reading, MA, 1999.

[6] C. Krattenthaler, Lattice path enumeration, in: Handbook of enumerative combinatorics, Discrete Math. Appl. (Boca Raton), pp. 589-678. CRC Press, Boca Raton, FL, 2015.

[7] H. MularczyK, Lattice paths and pattern-avoiding uniquely sorted permutations, at https://arxiv.org/abs/1908.04025.

[8] E. Pergola, Two bijections for the area of Dyck paths, Discrete Math., 241 (2001) 435-447.

[9] E. Pergola, R. Pinzani, S. Rinaldi And R. A. Sulanke A bijective approach to the area of generalized Motzkin paths, Adv. in Appl. Math., 28 (2002) 580-591.

[10] F. Petrov and A. Vershik, International mathematics competition: Day 2 problem 8 , at /imc-math.ddns.net/pdf/imc2018-day2-questions.pdf.

[11] H. Prodinger, S. J. Selkirk And S. Wagner, On two subclasses of Motzkin paths and their relation to ternary trees, in: V. Pillwein and C. Schneider (Eds.), Algorithmic Combinatorics Enumerative Combinatorics, Special Functions and Computer Algebra in honour of Peter Paule on his 60th birthday, Springer, 2020, pp. 297-316.

[12] R. Vidunas, A generalization of Clausen's identity, Ramanujan J., 26 (2011) 133-146. 